\title{
Suppression of Dilepton Production at Finite Baryon Density
}

\author{
A. Dumitru, D. H. Rischke, Th. Schönfeld, L. Winckelmann, H. Stöcker, and W. Greiner \\ Institut für Theoretische Physik der J. W. Goethe Universität, Postfach 111932, D-6000 Frankfurt am Main II, Germany
}

(Received 20 April 1992; revised manuscript received 29 January 1993)

\begin{abstract}
We study dilepton production from a quark-gluon plasma of given energy density at finite quark chemical potential $\mu$ and find that the dilepton production rate is a strongly decreasing function of $\mu$. Therefore, the signal to background ratio of dileptons from a plasma created in a heavy-ion collision may decrease significantly.
\end{abstract}

PACS numbers: $25.75 .+\mathrm{r}, 12.38 . \mathrm{Mh}, 24.85 .+\mathrm{p}$

Quantum chromodynamics (QCD) is nowadays believed to be the fundamental theory of strong interactions. Latttice QCD calculations [1] exhibit a transition from normal nuclear matter to a phase of deconfined quarks and gluons, the so-called quark-gluon plasma (QGP). Presumably the early Universe was in this state up to about $10 \mu \mathrm{sec}$ after the big bang. Today one hopes to achieve sufficiently high energy densities to again create the QGP in heavy-ion collision experiments.

The plasma (if created) emits lots of particles. Among these, electromagnetic probes can leave the plasma volume without further interactions (due to their large mean free path) and are therefore especially suitable to carry information about its existence and properties (temperature, energy, density, etc.). Thus, the production of real and virtual photons (the latter can be detected as dileptons, e.g., $e^{+} e^{-}, \mu^{+} \mu^{-}$) is regarded as a possible signature for the QGP [2-5]. Many attempts are made to measure these particles in heavy-ion collision experiments [6].

Up to now calculations of the dilepton production rate $[2,3,5]$ neglected the baryo-chemical potential $\mu_{B}$ in the plasma. Then, the dilepton emission rate depends only on the plasma temperature. This temperature is related to the energy density $\epsilon$ by the Stefan-Boltzmann law $\epsilon \sim T^{4}$, if $\mu_{B}=0$. The energy density of the QGP created in a collision can be readily estimated from the measured transverse energy and geometrical considerations $[7,8]$ or HBT measurements [9] of the plasma volume. This, in turn, allows the calculation of the total dilepton production rate from experimentally measurable quantities, without any assumptions of a specific dynamical model.

However, experiments [10] and theory [11] indicate that up to CERN SPS energies a sizable amount of baryon stopping occurs, i.e., the Bjorken scenario [12] seems not to be realistic for heavy-ion collisions at these "moderate" energies. In fact, even at RHIC bombarding energies $\sqrt{s} \leq 200 \mathrm{~A} \mathrm{GeV}$ recent calculations using microscopic models [13] hint that the colliding heavy ions may not be fully transparent. Consequently, it is possible that the baryon density (and thus $\mu_{B}$ ) in the QGP does not vanish. In this case the dilepton production rate is, in (local) thermodynamical equilibrium, a function of both temperature $T$ and quark chemical potential $\mu$ of the
QGP (we consider only $u$ and $d$ quarks, and therefore $\mu=\mu_{B} / 3$ ). In the present Letter we study the influence of such nonvanishing quark chemical potential $\mu$ on the dilepton production.

Since the Stefan-Boltzmann relation $\epsilon \sim T^{4}$ does not hold for $\mu / T>0$, we need to specify an equation of state (EOS) for the plasma which relates $\epsilon, T$, and $\mu$. Since we lack a self-consistent EOS derived from QCD, we use the phenomenological MIT-bag-model EOS [14], which yields the energy density

$$
\epsilon=\frac{37 \pi^{2}}{30} T^{4}+3 T^{2} \mu^{2}+\frac{3}{2 \pi^{2}} \mu^{4}+B
$$

and the baryon density

$$
n_{B}(\mu, T)=\frac{1}{3} \frac{\partial p(\mu, T)}{\partial \mu}=\frac{2}{3} \mu\left(T^{2}+\mu^{2} / \pi^{2}\right),
$$

where $p=(\epsilon-4 B) / 3$ is the pressure and $B=(235 \mathrm{MeV})^{4}$ [15]. We use this EOS (1) to calculate $T$ and $\mu$ for a given $\epsilon$ and $\mu / T$. As already stressed above, the energy density in a heavy-ion collision can be estimated without any model assumptions directly from experimental data, but not the temperature (temperatures derived from particle spectra require the assumption of a thermal source). In our calculations we use energy densities between 6 and $10 \mathrm{GeV} / \mathrm{fm}^{3}$, where one expects to be well in the QGP phase. This is well above the commonly assumed critical energy density of $1-2 \mathrm{GeV} / \mathrm{fm}^{3}[16]$.

A rough estimate of the value of $\mu / T$ in heavy-ion collisions may be obtained in the following way. As a first approximation, we will assume that the QGP is in thermal and chemical equilibrium. If two quark flavors are considered, the total pion multiplicity per rapidity is related to the initial temperature $T_{0}$ and the formation time $\tau_{0}$ by (see Ref. [17])

$$
\frac{d N_{\pi}}{d y}(y=0)=\frac{40}{\pi^{2}} T_{0}^{3} \tau_{0} \pi R_{A}^{2}
$$

where $R_{A}$ is the radius of the nuclei (note that this equation holds only at midrapidity). From Eq. (2) it follows that

$$
\frac{d N_{B}}{d z}=\frac{2}{3}\left(\mu T^{2}+\mu^{3} / \pi^{2}\right) \pi R_{A}^{2} .
$$

Using 1D ideal scaling hydrodynamics [12], we obtain a 
cubic equation for $\mu / T$, if we divide (4) by (3):

$$
\left(\frac{\mu}{T}\right)^{3}+\pi^{2} \frac{\mu}{T}=60 \cosh y \frac{d N_{B}}{d y} / \frac{d N_{\pi}}{d y}(y=0) .
$$

On the right-hand side of Eq. (5) we use the baryon and pion rapidity distributions of Ref. [13]. The resulting $\mu / T$ is shown in Fig. 1. The full line indicates the $\mu / T$ values in the early stage of the collision, where the QGP might exist; according to Eq. (5), $\mu / T$ is larger since less pions are produced at earlier times. A more detailed discussion will be given elsewhere [18]. We find that $\mu / T$ is indeed quite small at midrapidity. However, $\mu / T$ increases rapidly with rapidity: In the region $|y|$ $>1, \mu \approx T$. In fact, this is the interesting region for the proposed RHIC dilepton experiments, since they are not able to measure at $y \approx 0$ [19]. This crude estimate shows that $\mu$ may be of the same order of magnitude as $T$ (at least in the experimentally interesting region) even at RHIC.

The dilepton production rate $d N / d^{4} X d^{4} P \equiv d R / d^{4} P$ (i.e., the number of dileptons produced per space-time volume and four-dimensional momentum-space volume) is given, to $O\left(\alpha^{2}\right)$, by

$$
\frac{d R}{d^{4} P}=\int \frac{d^{3} \mathbf{k}_{1}}{(2 \pi)^{3}} \frac{d^{3} \mathbf{k}_{2}}{(2 \pi)^{3}} N_{F}\left(k_{1} ; T, \mu\right) N_{F}\left(k_{2} ; T,-\mu\right)_{v_{q \bar{q}}} \sigma_{q \bar{q}}^{l^{+} l^{-}} \delta^{(4)}\left(P-k_{1}-k_{2}\right),
$$

where $N_{F}$ is the Fermi-Dirac distribution function, $v_{q \bar{q}}$ is the relative velocity between quark and antiquark, and $\sigma_{q \bar{q}}^{l+l^{-}}$is the total cross section for the reaction $q \bar{q} \rightarrow l^{+} l^{-}$(summed over all initial states):

$$
\sigma_{q \bar{q}}^{l^{+l^{-}}}=\frac{5}{9} \frac{16 \pi \alpha^{2}}{M^{2}}\left(M^{2}=P^{\mu} P_{\mu} \text { is the invariant dilepton mass }\right) \text {, }
$$

where quark and lepton masses are neglected. Equation (6) yields a dilepton rate of

$$
\frac{d R}{d^{4} P}=\frac{5 \alpha^{2}}{36 \pi^{4}} \frac{T}{p} \frac{1}{e^{E / T}-1} \ln \left[\frac{\cosh ^{2}[(p+E) / 4 T]-\tanh ^{2}(\mu / 2 T) \sinh ^{2}[(p+E) / 4 T]}{\cosh ^{2}[(p-E) / 4 T]-\tanh ^{2}(\mu / 2 T) \sinh ^{2}[(p-E) / 4 T]}\right],
$$

which coincides with the result of Ref. [4] and that of Ref. [5] for $\mu=0$.

Figure 2(a) shows $d R / d^{4} P$ as given by (7) for large-mass pairs $(M=3 \mathrm{GeV})$ as a function of the absolute value of the lepton pair three-momentum $p=|\mathbf{P}|$ for $\epsilon=8 \mathrm{GeV} / \mathrm{fm}^{3}$ and different values of $\mu / T$. Observe that the rate decreases drastically for increasing $\mu / T$, due to the fact that the antiquark population is strongly suppressed for $\mu / T>0$ : Only few quark-antiquark pairs exist which may annihilate to form a $l^{+} l^{-}$pair. For dileptons with low invariant mass the next-order processes $\left[O\left(\alpha^{2} \alpha_{S}\right)\right]$ become important [20]. These are calculated below.

In Fig. 3 we show the mass spectrum $d R^{l^{+} l^{-}} / d M$ resulting from Eq. (7) by an appropriate change of variables. For a mass of, e.g., $M=3 \mathrm{GeV}$, dilepton production is suppressed by a factor of 14 if $\mu / T=2$ as compared to the case of $\mu / T=0$. This suppression factor rises with increasing mass.

By simply omitting the $\delta$ function in the rate integral (6), we obtain the total rate $R \equiv d N / d^{4} X$, i.e., the number of dileptons per space-time volume element,

$$
R=\frac{10 \alpha^{2} T^{4}}{9 \pi^{3}}\left[\frac{\pi^{4}}{144}-I^{2}-2 \frac{\mu}{T} I \ln \left(1+e^{\mu / T}\right)+\frac{\mu^{2}}{T^{2}}\left(\frac{\pi^{2}}{24}+\frac{3}{2} I-\ln ^{2}\left(1+e^{\mu / T}\right)\right]+\frac{3}{2} \frac{\mu^{3}}{T^{3}} \ln \left(1+e^{\mu / T}\right)-\frac{1}{2} \frac{\mu^{4}}{T^{4}}\right],
$$

with

$$
I=\int_{0}^{\mu / T} \frac{x d x}{e^{x}+1}=\frac{\pi^{2}}{12}-\frac{\mu}{T} \ln \left(1+e^{-\mu / T}\right)-\sum_{n \geq 1} \frac{(-1)^{n+1}}{n^{2}} e^{-n \mu / T} .
$$

Note that, for $\mu=0, R=\left(10 \alpha^{2} T^{4} / 9 \pi^{3}\right) \pi^{4} / 144$, the additional factor $\pi^{4} / 144$ in comparison to the result of Ref. [3] is due to the fact that we do not employ the Boltzmann approximation but use full quantum statistical distribution functions. The $T$ dependence can easily be understood from dimensional arguments considering Eq. (6) (without the $\delta$ function). In Fig. 4 we show $R$ as a function of $\mu / T$ for various energy densities. Observe that the total rate decreases rapidly with increasing $\mu / T$. 
For dileptons with low invariant mass the next-order processes become important [20]. However, as we will discuss now, the dilepton suppression at finite $\mu / T$ still occurs [see Fig. 2(b)]. We consider the contributions from the QCD annihilation $\left(q+\bar{q} \rightarrow g+l^{+} l^{-}\right)$and Compton $\left(q+g \rightarrow q+l^{+} l^{-}, \bar{q}+g \rightarrow \bar{q}+l^{+} l^{-}\right)$reactions to the dilepton production rate to $O\left(\alpha^{2} \alpha_{S}\right)$. The contribution from each of these reactions to the dilepton production rate is given by

$$
\frac{d R_{i}}{d^{4} P}=\frac{1}{(2 \pi)^{8}} \int \frac{d^{3} \mathbf{k}_{1}}{2 E_{1}} \frac{d^{3} \mathbf{k}_{2}}{2 E_{2}} \frac{d^{3} \mathbf{k}_{3}}{2 E_{3}} N_{1} N_{2}\left(1 \pm N_{3}\right) \delta^{(4)}\left(k_{1}+k_{2}-k_{3}-P\right)\left|M_{i}\right|^{2} .
$$

$N_{j}=N_{j}\left(k_{j} ; T, \mu\right)$ are the particle distribution functions. The sign in front of $N_{3}$ is chosen according to Bose enhancement or Pauli suppression for the strongly interacting final-state particles and $\mathcal{M}_{i}$ is the amplitude for the corresponding reaction. Assuming massless quarks we have

$$
\left|M_{\mathrm{ann}}\right|^{2}=\frac{5}{9} \frac{\pi 2^{9} \alpha^{2} \alpha_{S}}{3 M^{2}} \frac{u^{2}+t^{2}+2 s M^{2}}{u t},\left|M_{\text {Comp }, i}\right|^{2}=\frac{5}{9} \frac{\pi 2^{9} \alpha^{2} \alpha_{S}}{3 M^{2}} \frac{u^{2}+s^{2}+2 t M^{2}}{-u s},
$$

where $s, t, u$ are the Mandelstam variables. The index $i$ denotes either the $q$ - or the $\bar{q}$-Compton reaction, respectively. Applying the Boltzmann approximation for the incoming particles, i.e., restricting ourselves to high-energy dileptons with $E \gg T+\mu$, we arrive at

$$
\frac{d R_{\mathrm{ann}}}{d^{4} P}=\frac{1}{(2 \pi)^{8}} \frac{\pi^{2}}{4} \frac{T}{p} e^{-E / T} \int_{M^{2}}^{\infty} d s \frac{1}{s-M^{2}} \ln \left(\frac{1-e^{-\left(s-M^{2}\right) / 2 T(E-p)}}{1-e^{-\left(s-M^{2}\right) / 2 T(E+p)}}\right) \int_{M^{2}-s}^{0} d t\left|M_{\mathrm{ann}}\right|^{2}
$$

for the annihilation and

$$
\frac{d R_{\mathrm{Comp}, i}}{d^{4} P}=\frac{1}{(2 \pi)^{8}} \frac{\pi^{2}}{4} \frac{T}{p} e^{-E / T} \int_{M^{2}}^{\infty} d s \frac{1}{s-M^{2}} \ln \left(\frac{e^{\mp \mu / T}-e^{\left(s-M^{2}\right) / 2 T(E+p)}}{e^{\mp \mu / T}-e^{-\left(s-M^{2}\right) / 2 T(E-p)}}\right) \int_{M^{2}-s}^{0} d t\left|M_{\mathrm{Comp}, i}\right|^{2}
$$

for the Compton contributions (the upper sign is for the $q-$, the lower one for the $\bar{q}$-Compton reaction, and again

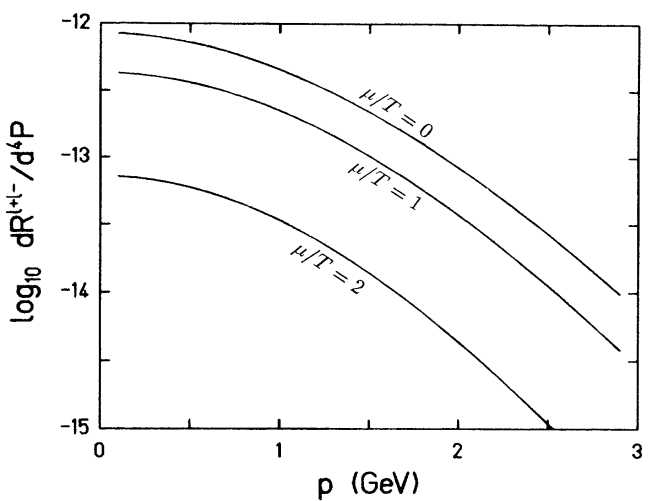
$p=|\mathbf{P}|$ ). These contributions alone exhibit collinear singularities (the $t$ integration is infrared divergent due to the fact that the quarks are considered as massless), which vanish, if self-energy and vertex corrections are taken into account [21]. The evaluation of the corresponding diagrams is laborious [20] and, for our case (arbitrary $\mu$ ), out of the scope of the present Letter. To estimate the size of this effect one can regularize the rate in a similar manner as the photon production rate in Ref. [22]. As a result of in-medium effects, a thermal quark mass $m_{f}^{2}=g^{2}\left(T^{2}+\mu^{2} / \pi^{2}\right) / 6$ (see [22,23]) acts as a cutoff at low momentum transfer. The integration boundaries change to $M^{2}+4 m_{f}^{2} \leq s<\infty$ and $M^{2}+2 m_{f}^{2}-s \leq t$

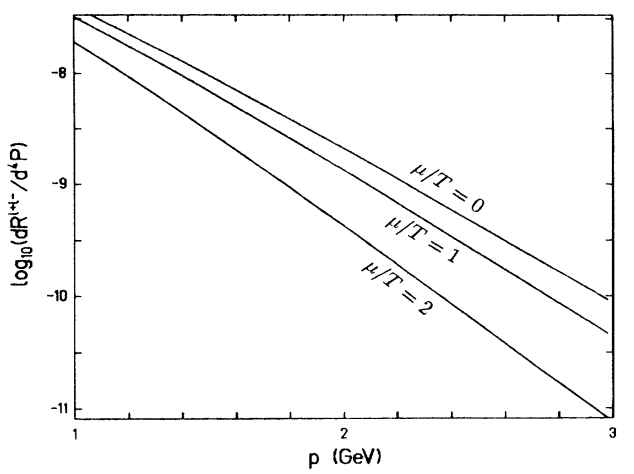

FIG. 2. (a) The rate $d R / d^{4} P$ to $O\left(\alpha^{2}\right)$ at energy density $\epsilon=8 \mathrm{GeV} / \mathrm{fm}^{3}$ for $\mu / T=2,1,0$; the dilepton invariant mass is taken as $M=3 \mathrm{GeV}$. (b) The rate $d R / d^{4} P$ to $O\left(\alpha^{2} \alpha_{S}\right)$ at energy density $\epsilon=8 \mathrm{GeV} / \mathrm{fm}^{3}$ for $\mu / T=2,1,0$; the dilepton invariant mass is taken as $M=0.1 \mathrm{GeV}$.

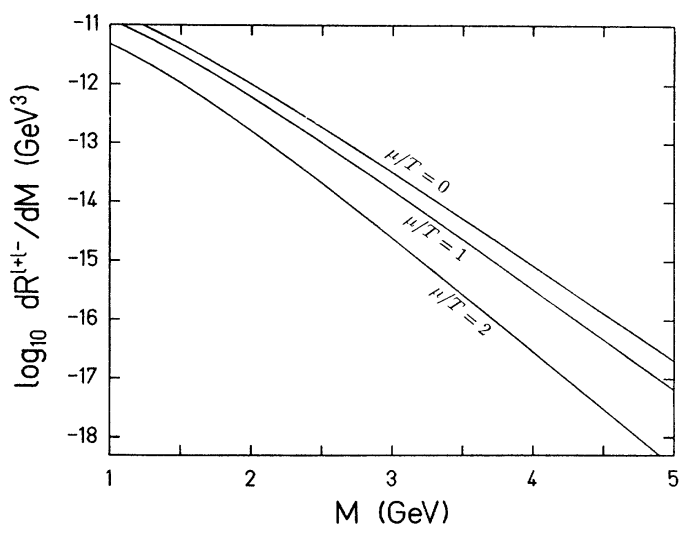

FIG. 3. The rate per mass interval $d R^{I^{+} I^{-}} / d M$ at energy density $\epsilon=8 \mathrm{GeV} / \mathrm{fm}^{3}$ for $\mu / T=2,1,0$. 


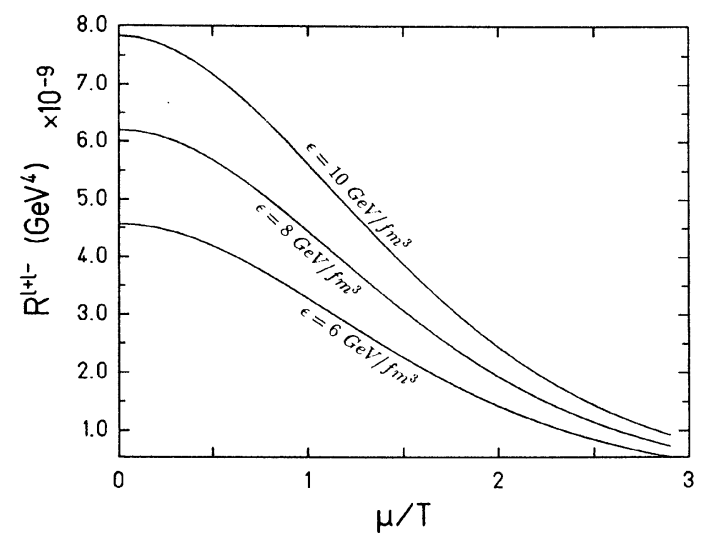

FIG. 4. The total rate $R$ for $\epsilon=6,8,10 \mathrm{GeV} / \mathrm{fm}^{3}$.

$\leq-2 m_{f}^{2}$ [22]. Now the $t$ integration can be done and the remaining $s$ integration may be performed numerically. In Fig. 2(b) the resulting dilepton production rate is shown for different values of the quark chemical potential $\mu / T$ and a dilepton mass of $M=0.1 \mathrm{GeV}$. As already mentioned, the rate again drops with increasing $\mu / T$, e.g., at momentum $p=2 \mathrm{GeV}$ there is a dilepton suppression by a factor of 5 if $\mu / T=2$ as compared to the case $\mu / T=0$. Thus, the predicted suppression of dilepton production can be expected to hold also to $O\left(\alpha^{2} \alpha_{s}\right)$. Nevertheless, it is not as strong as in Fig. 2(a); low mass dileptons $(M \approx 0.1 \mathrm{GeV})$ are somewhat less sensitive to finite chemical potential effects. However, it is difficult, if not impossible, to observe the low mass dileptons due to a huge background of hadronic Dalitz decays.

In conclusion, we have demonstrated that dilepton production is suppressed by factors of 3-10 (depending on the value of $\mu / T$, the dilepton mass and momentum, the plasma energy density, etc.) as compared to previous estimates for a baryon-free plasma (i.e., $\mu / T=0$ ). This prediction of dilepton suppression is important for several large-scale experiments at SPS and RHIC.

[1] M. Creutz, Quarks, Gluons and Lattices (Cambridge Univ. Press, Cambridge, 1983); Y. Deng, Nucl. Phys. (Proc. Suppl.) B9 334 (1989); F. R. Brown, N. H. Christ, Y. Deng, M. Gao, and T. J. Woch, Phys. Rev. Lett. 61, 2058 (1988); J. Engels, J. Fingberg, F. Karsch, D. Miller, and M. Weber, Phys. Lett. B 252, 625 (1990).

[2] See, for instance, L. D. McLerran and T. Toimela, Phys. Rev. D 31, 545 (1985); M. Neubert, Z. Phys. C 42, 231 (1989); R. Baier, H. Nakkagawa, A. Niégawa, and K. Redlich, Z. Phys. C 53, 433 (1992), and references therein.

[3] K. Kajantie, J. Kapusta, L. McLerran, and A. Mekjian, Phys. Rev. D 34, 2746 (1986).
[4] J. Cleymans, J. Fingberg, and K. Redlich, Phys. Rev. D 35, 2153 (1987).

[5] M. I. Gorenstein and O. A. Mogilevsky, Phys. Lett. B 228, 121 (1989).

[6] See, for example, G. Roche, The Nuclear Equation of State, Peñiscola, Spain, 1989, NATO Advanced Study Institutes, Ser. A, Vol. 261 (Plenum, New York), p. 161; HELIOS Collaboration, J. Schukraft et al., Nucl. Phys. A498, 79c (1989); P. Braun-Munzinger and G. David, in Proceedings of the Twentieth International Workshop on Gross Properties of Nuclei and Nuclear Excitations, Hirschegg, Austria, 1992, edited by H. Feldmeier.

[7] NA35 Collaboration, W. Heck et al., Z. Phys. C 38, 19 (1988).

[8] NA35 Collaboration, J. W. Harris et al., Nucl. Phys. A498, 133c (1989).

[9] T. Humanic, Z. Phys. C 38, 79 (1988).

[10] W. Busza, Nucl. Phys. A418, 635c (1984); O. Hansen, in Proceedings of the Twentieth International Workshop on Gross Properties of Nuclei and Nuclear Excitations, Hirschegg, Austria, 1992, edited by H. Feldmeier; NA35 Collaboration, Report No. LBL-27833, 1989 (unpublished); NA35 Collaboration, G. Odyniec et al., Proceedings of the International Workshop on Relativistic Aspects of Nuclear Physics, Rio de Janeiro, 1989.

[11] A. v.Keitz, L. Winckelmann, A. Jahns, H. Sorge, H. Stöcker, and W. Greiner, Phys. Lett. B 263, 355 (1991); H. Sorge, A. v.Keitz, R. Matiello, H. Stöcker, and W. Greiner, Phys. Lett. B 243, 7 (1990).

[12] J. D. Bjorken, Phys. Rev. D 27, 140 (1983).

[13] T. Schönfeld, H. Sorge, H. Stöcker, and W. Greiner, UFTP Report No. 314/1992 (to be published); G. Gustafson, Proceedings of the Workshop on Relativistic Heavy-Ion Physics at Present and Future Accelerators, Budapest, 1992; H. J. Möhring and J. Ranft, Z. Phys. C 52, 643 (1991).

[14] A. Chodos, R. I. Jaffe, K. Johnson, C. B. Thorn, and V. Weisskopf, Phys. Rev. D 9, 347 I (1974).

[15] B. M. Waldhauser, D. H. Rischke, J. A. Maruhn, H. Stöcker, and W. Greiner, Z. Phys. C 43, 41 I (1989).

[16] B. Müller, The Physics of the Quark-Gluon Plasma (Springer, Berlin, 1985).

[17] R. Hwa and K. Kajantie, Phys. Rev. D 32, 1109 (1985); J. Kapusta, L. McLerran, and D. Srivastava, University of Minnesota Report No. TPI-MINN-92/3-T (to be published).

[18] A. Dumitru, D. H. Rischke, H. Stöcker, W. Greiner, and L. McLerran, UFTP Report No. 314 (to be published).

[19] Phoenix Collaboration, S. Nagamia et al., GSI, 1992.

[20] T. Altherr and P. V. Ruuskanen, University of Jyväskylä Report No. JYFL 7/1992 (to be published).

[21] R. Baier, B. Pire, and D. Schiff, Phys. Rev. D 38, 2814 (1988); P. Altherr, P. Aurenche, and T. Becherrawy, Nucl. Phys. B315, 436 (1989).

[22] J. Kapusta, P. Lichard, and D. Seibert, Phys. Rev. D 44, 2774 (1991).

[23] K. Kajantie and P. V. Ruuskaanen, Phys. Lett. 121B, 352 (1983). 